\title{
Adrenostatische Therapie mit Metyrapon und Aminoglutethimid beim ACTH-abhängigen Cushing- Syndrom
}

\author{
M. Reincke, B. Allolio, C. Jaursch-Hancke, D. Kaulen und W. Winkelmann \\ Medizinische Universitãtsklinik II, Köln-Merheim (Direktor: Prof. Dr. W. Kaufmann)
}

Bei zehn Patienten mit ACTH-abhängigem CushingSyndrom, vier mit ektopem Cushing-Syndrom und sechs mit M. Cushing, wurde retrospektiv die Wirksamkeit einer adrenostatischen Therapie mit Metyrapon (Metopiron ${ }^{\circledR}$ ) und Aminoglutethimid (Orimeten $\left.{ }^{\circledR}\right)$ untersucht. Unter Metyrapon allein $(n=5)$ sowie in Kombination mit Aminoglutethimid $(n=5)$ kam es bei allen Patienten zu einer dauerhaften Senkung der Serum-Cortisol-Konzentration. Die Beobachtungszeit betrug 2 Wochen bis 4 Jahre. Der angestrebte therapeutische Bereich von $<16 \mu \mathrm{g} / \mathrm{dl}$ wurde bei sieben Patienten erreicht. Im Verlauf der Therapie wurde ein Anstieg der mittleren Plasma-ACTH-Konzentration beobachtet; dabei kam es nicht zu einem "Escape " der Cortisol-Konzentration. Eine Einschränkung erfuhr die adrenostatische Therapie allein durch die Nebenwirkungen, die bei zwei Patienten zu einer Beendigung der Therapie führten. Folgerung: Die adrenostatische Therapie mit Metyrapon und Aminoglutethimid ist wirksam und praktikabel. Sie eignet sich nicht nur zur akuten Behandlung des floriden Cushing-Syndroms, sondern auch zur Langzeittherapie, wenn eine kurative Therapie nicht möglich ist.
Adrenostatic treatment of ACTH-dependent Cushing's syndrome with metyrapone and aminoglutethimide

The adrenostatic effect of metyrapone (Metopiron ${ }^{\circledR}$ ) and aminoglutethimide (Orimeten ${ }^{\circledR}$ ) was assessed retrospectively in ten patients with ACTH-dependent Cushing's syndrome, four of them with the ectopic form. Five patients received metyrapone only, the other five both metyrapone and aminoglutethimide. Persistent lowering of the serum cortisol level was achieved in all, after an observation period of two weeks to four years. The intended therapeutic level of below $16 \mu \mathrm{g} / 100 \mathrm{ml}$ was achieved in seven patients. In the course of treatment there was a rise in mean plasma ACTH concentration, but without an "escape " phenomenon. The adrenostatic treatment had to be limited, if at all, only because of side effects, which in two patients required that the drug administration be terminated. It is concluded that adrenostatic treatment with metyrapone and aminoglutethimide is effective and practical. It is suitable not only in the management of florid Cushing's syndrome, but also for long-term treatment when complete cure is not possible.
Das unbehandelte Cushing-Syndrom ist mit einer hohen Morbidität belastet. Der exzessive Hypercortisolismus, wie er besonders für das ektope Cushing-Syndrom charakteristisch ist, führt zu ausgeprägten Krankheitssymptomen wie Abgeschlagenheit, Muskelschwäche, arteriellem Hypertonus, Hypokaliämien und psychotischen Zustandsbildern und bedarf deshalb einer schnellen Behandlung (6). Wegen eines erhöhten Risikos von thromboembolischen Komplikationen, Blutungen und Wundheilungsstörungen (13) ist vor definitiven therapeutischen Maßnahmen (transsphenoidale Hypophysenoperation, Adrenalektomie) eine medikamentöse Kontrolle des Hypercortisolismus sinnvoll $(7,14)$. Mit Metyrapon (Metopiron ${ }^{\circledR}$ ) und Aminoglutethimid (Orimeten ${ }^{\circledR}$ ) stehen zwei Substanzen zur Verfügung, die zu einer

Dtsch. med. Wschr. 112 (1987), 1532-1535

(c) 1987 Georg Thieme Verlag Stuttgart - New York reversiblen Blockade der Steroidsynthese in der Nebenniere führen (2).

Wir berichten im folgenden über unsere Erfahrungen mit einer adrenostatischen Therapie mit Metyrapon allein oder in Kombination mit Aminoglutethimid beim $\mathrm{ACTH}$-abhängigen Cushing-Syndrom. $\mathrm{Zu}$ beantworten waren dabei die Fragen, ob es unter dieser Therapie zu einer dauerhaften Senkung der Serum-Cortisol-Konzentration kommt und welche Gründe zu einer Beendigung der adrenostatischen Therapie zwingen.

\section{Patienten und Methoden}

Patienten. Von 1982 bis 1986 wurden zehn Patienten mit ACTHabhängigem Cushing-Syndrom betreut (Tabelle 1). Bei sechs Patienten bestand ein zentrales Cushing-Syndrom (M. Cushing), vier Patienten wiesen eine ektope ACTH-Sekretion auf (eine Patientin mit medullärem Schilddrüsenkarzinom, ein Patient mit kleinzelligem Bronchialkarzinom, ein Patient mit metastasierendem Karzinoid-Syndrom, eine Patientin mit okkultem ektopem Cushing- 
Syndrom). Die der adrenostatischen Behandlung vorausgegangenen erfolglosen Therapieversuche bei den M.-Cushing-Patienten bestanden in dreimaliger transsphenoidaler Hypophysenoperation (Fall 5), transsphenoidaler Hypophysenoperation und Bestrahlung (Fall 6), transsphenoidaler Hypophysenoperation (Fall 7 und 9), und Hypophysenbestrahlung (Fall 10). Bei einem Patienten (Fall 8) war keine Therapie vorausgegangen.

Ein Cushing-Syndrom wurde diagnostiziert mittels Standardkriterien (6) bei erhöhtem Serum-Cortisolspiegel, Fehlen des zirkadianen Rhythmus der Cortisolsekretion, erhöhtem freiem Cortisol im 24-Stunden-Urin und fehlender Suppression des Serum-Cortisols im Dexamethason-Kurztest. Mittels hochdosierter Dexamethasongabe, Plasma-ACTH-Messung, CRH-Test (100 $\mu \mathrm{g}$ CRH intravenös), Lysin-Vasopressin-Test (5 IU in 60 Minuten intravenös), selektiver Venenblutentnahme sowie radiologischer Verfahren (Computertomographie usw.) wurde zwischen zentralem CushingSyndrom, Nebennierenadenom bzw. -karzinom und ektoper ACTH-Sekretion differenziert.

Methodik. Die Bestimmung von ACTH im Plasma erfolgte radioimmunologisch (1) nach Extraktion mit Quso $G_{32}(16)$. Wir verwendeten als Standard $\beta^{1-39}$-ACTH des Medical Research Council (74/555) (Normalwert $<50 \mathrm{pg} / \mathrm{ml}$ um 9 Uhr). Serum-Cortisol wurde radioimmunologisch mit im Handel erhältlichen Reagenzien (NEN, Dreieich) gemessen (Normalbereich 5-25 $\mu \mathrm{g} / \mathrm{dl}$ um $9 \mathrm{Uhr}$ ).

Therapieprotokoll. Nach Diagnosestellung wurde eine adrenostatische Therapie mit Metyrapon eingeleitet. Die Anfangsdosierung betrug dreimal $250 \mathrm{mg} / \mathrm{d}$ und wurde in Abhängigkeit von der Wirkung gesteigert. Fünf Patienten erhielten zusätzlich Aminoglutethimid bis zu einer Gesamtdosis von $2 \mathrm{~g} / \mathrm{d}$ (Tabelle 1). Die Therapie wurde reduziert oder abgebrochen bei erheblichen Nebenwirkungen (Übelkeit, Erbrechen, Müdigkeit), Erfolglosigkeit oder bei Durchführung definitiver therapeutischer Maßnahmen (zum Beispiel Operation).

Die Therapie wurde überwacht durch Kurzprofile von PlasmaACTH und Serum-Cortisol über 3 Stunden vor Behandlungsbeginn und in regelmäßigen Abständen während der Behandlung. Diese Messungen wurden ergänzt durch Kontrollen von Serum-Kalium, Blutzucker und klinischer Beurteilung (Gewichtsentwicklung, Rückbildung von Cushing-Stigmata usw.). Die Krankheitsgeschichten wurden retrospektiv ausgewertet.

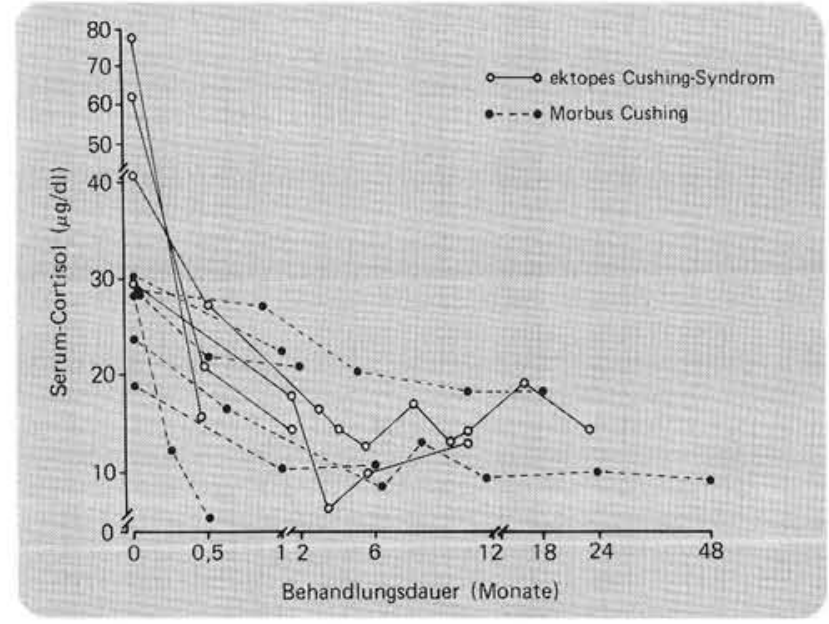

Abb. 1. Verlauf der Serum-Cortisol-Konzentration bei Patienten mit $\mathrm{ACTH}$-abhängigem Cushing-Syndrom während Behandlung mit Metyrapon und Aminoglutethimid.

\section{Ergebnisse}

Bei allen Patienten kam es unter dem Einfluß der Behandlung zu einem Absinken der Serum-Cortisol-Konzentration (Tabelle 1, Abbildung 1). Der angestrebte therapeutische Bereich von $<16 \mu \mathrm{g} / \mathrm{dl}$ wurde bei sieben der zehn Patienten erreicht. Bei den drei übrigen Patienten konnte wegen Nebenwirkungen keine ausreichende Dosissteigerung vorgenommen werden.

Bei keinem Patienten wurde während der Behandlung eine Substitutionstherapie erforderlich oder durchgeführt. Bei Absinken des Serum-Cortisols in den therapeutischen Bereich kam es zu einer deutlichen Besserung der klinischen Symptomatik. Schwere psychische Auffälligkeiten, die in zwei Fällen primär zur Aufnahme in einer Psychiatrischen Klinik geführt hatten, besserten sich

Tab. 1. Adrenostatische Langzeittherapie bei Patienten mit ACTH-abhängigem Cushing-Syndrom

\begin{tabular}{|c|c|c|c|c|c|c|c|c|}
\hline \multirow[b]{2}{*}{ Fall } & \multirow[b]{2}{*}{ Diagnose } & \multirow[b]{2}{*}{$\begin{array}{c}\text { Alter } \\
\text { (Jahre), } \\
\text { Geschlecht }\end{array}$} & \multirow[b]{2}{*}{$\begin{array}{c}\text { Metyrapon } \\
(\mathrm{g} / \mathrm{d})\end{array}$} & \multirow[b]{2}{*}{$\begin{array}{l}\text { Amino- } \\
\text { glutethimid } \\
(\mathrm{g} / \mathrm{d})\end{array}$} & \multicolumn{2}{|c|}{$\begin{array}{l}\text { Serum-Cortisol } \\
(\mu \mathrm{g} / \mathrm{dl})\end{array}$} & \multirow[b]{2}{*}{$\begin{array}{l}\text { Therapie- } \\
\text { dauer } \\
\text { (Monate) }\end{array}$} & \multirow[b]{2}{*}{ Bemerkungen } \\
\hline & & & & & $\begin{array}{l}\text { vor } \\
\text { Tr }\end{array}$ & $\begin{array}{l}\text { während } \\
\text { apie }\end{array}$ & & \\
\hline 1 & ektopes Cushing-Syndrom & $42 \sigma^{\circ}$ & 1,0 & 2,0 & 41,3 & 14,5 & 23 & zur Zeit noch behandelt \\
\hline 2 & ektopes Cushing-Syndrom & 38 ? & 1,0 & 1,0 & 95,5 & 15,2 & 0,5 & gestorben \\
\hline 3 & ektopes Cushing-Syndrom & $71 \delta$ & 1,0 & 1,0 & 62,4 & 14,4 & 1,5 & gestorben \\
\hline 4 & ektopes Cushing-Syndrom & $37 \%$ & $1,25-1,5$ & - & 29,4 & 14,3 & 11 & zur Zeit noch behandelt \\
\hline 5 & M. Cushing & 13 운 & 0,5 & 0,5 & 28,3 & 21,4 & 2,5 & zur Zeit noch behandelt \\
\hline 6 & M. Cushing & 41 ? & $0,75-1,0$ & - & 28,6 & 18,4 & 18 & bilaterale Adrenalektomie \\
\hline 7 & M. Cushing & 40 ? & 1,0 & 1,0 & 28,9 & 6,2 & 0,5 & bilaterale Adrenalektomie \\
\hline 8 & M. Cushing & $49 \%$ & $1,5-2,25$ & - & 23,7 & 9,1 & 48 & zur Zeit noch behandelt \\
\hline 9 & M. Cushing & 52 ? & 1,0 & - & 18,9 & 10,6 & 6 & $\begin{array}{l}\text { Therapieabbruch wegen } \\
\text { Nebenwirkungen }\end{array}$ \\
\hline 10 & M. Cushing & $53 \%$ & 1,0 & - & 30,4 & 22,6 & 3 & $\begin{array}{l}\text { Therapieabbruch wegen } \\
\text { Nebenwirkungen }\end{array}$ \\
\hline
\end{tabular}




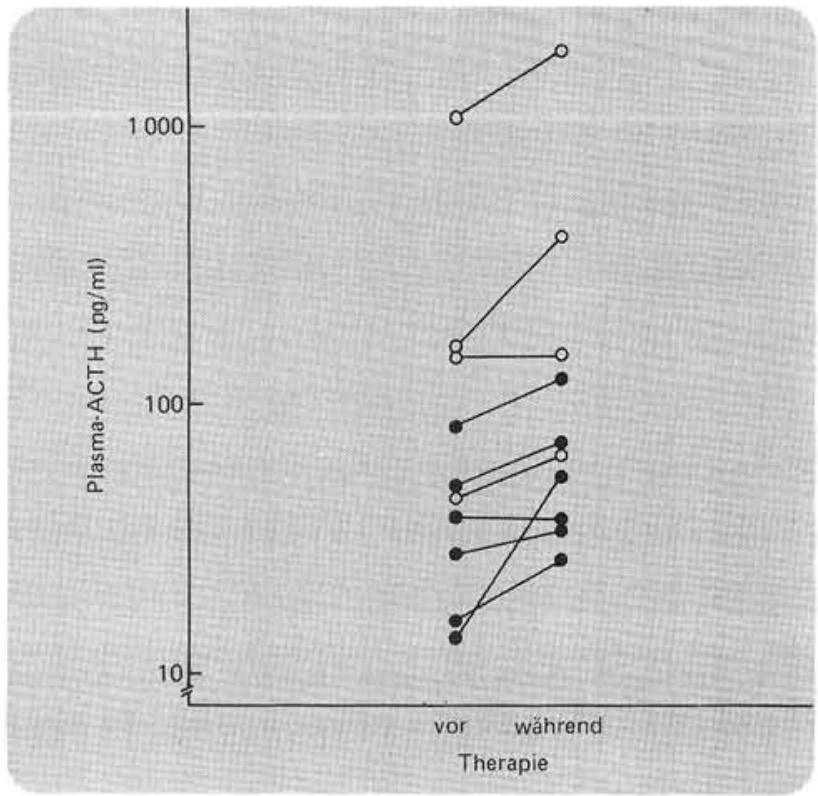

Abb. 2. Plasma-ACTH vor adrenostatischer Therapie beim ACTHabhängigen Cushing-Syndrom (offener Kreis=ektopes CushingSyndrom, geschlossener Kreis = M. Cushing) und maximale ACTHKonzentration während der Therapie (jeweils Mittelwerte von Profilen über 3 Stunden).

innerhalb weniger Tage. Eine anfangs vorhandene Hypokaliämie wurde ausgeglichen, ohne daß eine weitere Substitutionstherapie erforderlich wurde. Bei länger dauernder Therapie ( $>6$ Monate) kam es zu einer weitgehenden Rückbildung der Cushing-Stigmata wie Vollmondgesicht, Stammfettsucht und Rubeosis (Fall 1, 4, 8 und 9).

Im Verlauf der Behandlung wurde ein Anstieg der mittleren Plasma-ACTH-Konzentration beobachtet, der jedoch bei keinem Patienten zu einem "Escape" der Cortisol-Konzentrationen führte. Bei Patienten mit M. Cushing lag die mittlere ACTH-Konzentration vor der Therapie bei $38 \pm 12 \mathrm{pg} / \mathrm{ml}$ (Bereich 14-83 pg/ml), während der Therapie bei $58 \pm 16 \mathrm{pg} / \mathrm{ml}$ (Bereich $28-128 \mathrm{pg} / \mathrm{ml})$. Bei Patienten mit ektopem Cushing-Syndrom wurden vor der Behandlung Plasma-ACTH-Konzentrationen zwischen 46 und $1200 \mathrm{pg} / \mathrm{ml}$, während der adrenostatischen Therapie zwischen 66 und $2900 \mathrm{pg} / \mathrm{ml}$ gemessen (Abbildung 2). Dabei ging der ACTH-Anstieg bei den Patienten mit ektopem Cushing-Syndrom mit einer Tumorprogression einher, der zwei Patienten erlagen. Auch bei diesen Patienten war jedoch kein Nachlassen der Wirkung der adrenostatischen Therapie erkennbar. Drei der Patienten (Fall 1, 4 und 8) werden jetzt seit 1-4 Jahren behandelt; bei fortbestehendem Grundleiden befinden sie sich in weitgehender klinischer Remission.

Die Hauptnebenwirkungen, die bei zwei Patienten (Fall 9 und 10) zur Beendigung der Therapie und bei zwei weiteren (Fall 5 und 6) zu einer Einschränkung der einsetzbaren Dosis führten, waren Übelkeit und pathologische Müdigkeit. Eine Patientin (Fall 7) mit myokardialer Insuffizienz wies eine vermehrte Ödemneigung auf. Eine Zunahme von Hirsutismus oder Akne trat nicht auf.

\section{Diskussion}

Die Therapie der Wahl beim Cushing-Syndrom besteht in der Beseitigung der Ursache des Hypercortisolismus (6). Beim zentralen Cushing-Syndrom ist dies in erster Linie die transsphenoidale Hypophysenoperation mit selektiver Adenomektomie (8). Beim Nebennierenadenom bzw. -karzinom wird die operative Entfernung des Tumors angestrebt (15). Das ektope Cushing-Syndrom ist dagegen aufgrund des meist fortgeschrittenen Grundleidens einer kurativen Therapie selten zugänglich, obwohl in günstigen Fällen eine komplette Resektion des ACTH-bildenden Tumors zu dauerhafter Heilung führen kann (5).

Neben anderen Therapieformen wie Hypophysenbestrahlung und beidseitiger Adrenalektomie stellt die medikamentöse Nebennierenblockade mit Mitotan (12), Ketoconazol (10) oder Metyrapon und Aminoglutethimid $(2,6,7,9,14)$ einen weiteren therapeutischen Ansatzpunkt dar. Die Möglichkeit einer dauerhaft wirksamen adrenostatischen Therapie ist jedoch bei Metyrapon und Aminoglutethimid in Frage gestellt worden in der Annahme, daß die Senkung der Cortisol-Konzentration zu einem kompensatorischen ACTH-Anstieg führt, der die Nebennierenblockade unwirksam werden läßt (4, 18). Die Ergebnisse unserer Untersuchung belegen jedoch die langfristige Wirksamkeit einer medikamentösen Nebennierenblockade. Mit Metyrapon allein oder in Kombination mit Aminoglutethimid kam es bei allen unseren Patienten zu einer dauerhaften Normalisierung der Serum-Cortisol-Konzentration. Dabei war der therapeutische Effekt unabhängig von der Höhe der ACTHKonzentration. Auch bei initial sehr hohen Werten (Fall 1-3) wurde eine rasche Senkung der Cortisol-Konzentration erreicht, die mit einer eindrucksvollen Besserung der akuten klinischen Symptomatik einherging. Bei keinem Patienten kam es während der Dauertherapie zu einem "Escape " der Cortisol-Konzentration, auch wenn ein Anstieg der ACTH-Konzentration zu verzeichnen war. $\mathrm{Da}$ im allgemeinen beim ektopen Cushing-Syndrom eine autonome, durch die Cortisol-Konzentration wenig beeinflußte ACTH-Sekretion vorliegt (6), ist der Anstieg bei diesen Patienten am chesten Folge der Tumorprogression. Der vermutlich kompensatorisch bedingte ACTHAnstieg beim M. Cushing war bei unseren Patienten dagegen nur gering ausgeprägt und ging nicht mit einem Wiederanstieg des Serum-Cortisols einher.

Diese Ergebnisse decken sich mit den Untersuchungen von Jeffcoate und Mitarbeitern (7) sowie Thoren und Mitarbeitern (14), die ebenfalls nur einen geringen ACTH-Anstieg beobachteten. Jedoch war in beiden Studien die Mehrzahl der Patienten mit M. Cushing vor der adrenostatischen Therapie einer Hypophysenbestrahlung zugeführt worden. Von unseren Patienten war hingegen nur eine Minderzahl vorbestrahlt, so daß eine strahlenbedingte Vorschädigung der Hypophyse als Erklärung für den geringen kompensatorischen ACTH-Anstieg entfällt. 
Interessanterweise wurde von einer Arbeitsgruppe sogar über eine Senkung der ACTH-Konzentration bei Behandlung mit Metyrapon berichtet (19), so daß eine direkte Wirkung des Mittels auf die ACTH-Sekretion angenommen wurde (14). Dieser Annahme stehen jedoch eigene Untersuchungen an Patienten mit M. Addison entgegen, bei denen bei Anwendung von Metyrapon keine Senkung der ACTH-Sekretion beobachtet wurde (17).

Die Anwendung von Metyrapon und Aminoglutethimid bietet somit eine wirksame Möglichkeit zur Langzeittherapie beim ACTH-abhängigen Cushing-Syndrom, die bei vier unserer Patienten erfolgreich über 6 Monate bis 4 Jahre eingesetzt wurde. Liegt ein ektopes CushingSyndrom vor, bei dem die Grundkrankheit einer kurativen Therapie nicht zugänglich ist, ist die adrenostatische Behandlung die Methode der Wahl (9). Das gilt auch dann, wenn sich die Ursache der ektopen ACTH-Sekretion einem raschen Nachweis entzieht, wie bei einer unserer Patientinnen (Fall 4). Durch die adrenostatische Therapie kann dabei Zeit gewonnen werden, bis der eigentliche Krankheitsherd ermittelt und beseitigt ist, ohne daß gleich eine bilaterale Adrenalektomie durchgeführt werden muß. Der Nachweis einer ACTH-Quelle bei einem solchen okkulten Cushing-Syndrom kann sich dabei über mehrere Jahre hinziehen (5). Beim zentralen Cushing-Syndrom läßt sich mit der adrenostatischen Therapie das klinische Befinden des Patienten erheblich bessern, so daß ein operatives Vorgehen zur definitiven Behandlung oft dann erst möglich wird. Schließlich kann diese Behandlung die Zeitspanne überbrücken, die bis zur Wirksamkeit einer Hypophysenbestrahlung verstreicht (7).

Eine Einschränkung erfährt die adrenostatische Therapie nicht durch ein Versagen oder Nachlassen der Wirksamkeit, sondern ausschließlich durch die Nebenwirkungen. Die Kombination von Metyrapon und Aminoglutethimid, wie sie in dieser Studie eingesetzt wurde, ist deswegen einer hochdosierten Monotherapie vorzuziehen, da sie mit weniger Nebenwirkungen behaftet ist (2) und durch die kombinierte Blockade der 11 $\beta$-Hydroxylase und der Desmolase (3) eine höhere Wirksamkeit besitzt. Schwere Nebenwirkungen treten unter Metyrapon allein selten auf (14). Subjektiv störende Nebenwirkungen wie Müdigkeit, Kreislaufbeschwerden und Übelkeit, wie wir sie bei vier Patienten beobachteten, sind jedoch relativ häufig. Sie zwangen bei zwei Patienten zur Beendigung der Therapie. Eine Verstärkung des Hirsutismus $(6,7)$ wurde bei keinem unserer Patienten gesehen. Von Aminoglutethimid werden als Nebenwirkungen vor allem Müdigkeit, Kopfschmerzen, Myalgien, Exantheme und gastrointestinale Beschwerden angegeben (14). Sie scheinen jedoch dosisabhängig zu sein und treten selten bei einer täglichen Dosierung unter $1 \mathrm{~g}$ auf (11). Wir beobachteten nur bei einer Patientin während kombinierter Therapie entsprechende Beschwerden (Übelkeit, Müdigkeit).

Zusammenfassend läßt sich feststellen, daß es sich bei der medikamentösen Nebennierenblockade mit Metyrapon und Aminoglutethimid beim ACTH-abhängigen Cushing-Syndrom um eine sichere und wirksame Therapie handelt. Eine Einschränkung erfährt die adrenostatische Therapie dabei nicht durch einen "Escape " der Cortisol-Konzentration, sondern ausschließlich durch die medikamentenbedingten Nebenwirkungen.

\section{Literatur}

(1) Allolio, B., W. Winkelmann, F. X. Hipp: Effect of meclastine, an $\mathrm{H}_{1}$-antihistamine, on plasma ACTH in adrenal insufficiency. Acta endocr. (Kbh.) 96 (1983), 98 .

(2) Child, D. F., C. W. Burke, D. W. Buricy, L. H. Rees, T. R. Fraser: Drug control of Cushing's syndrome. Acta endocr. (Kbh.) 82 (1975), 330 .

(3) Dexter, R. N., F. M. Lawrence, R. Ney, G. W. Liddle: Inhibition of adrenal corticosteroid synthesis by aminoglutethimid. Studies of the mechanism of action. J. clin. Endocr. Metab. 27 (1967), 473.

(4) Fehm, H. L.: Über- und Unterfunktion der Nebenniere. In Ziegler, R. (Hrsg.): Hormon- und stoffwechselbedingte Erkrankungen in der Praxis (Edition Medizin, Verlag Chemie: Weinheim 1987)

(5) Howlett, T. A., L. Drury, L. Perry, L. Doniach, L. H. Rees, G. M. Besser: Diag nosis and management of ACTH-dependent Cushing's syndrome: comparison of the features in ectopic and pituitary

$\mathrm{ACTH}$ production. Clin. Endocr. 24 (1986), 699.

(6) Howlett, T. A., L. H. Rees, G. M. Besser: Cushing's syndrome. Clin. in Endocr. Metab. 14 (1985), 911.

(7) Jeffcoate, W. J., L. H. Rees, S. Tomlin, A. E. Jones, C. R. W. Edwards, G. M Besser: Metyrapone in long-term management of Cushing's disease. Brit. med. J. $1977 / 2,215$.

(8) Krieger, D. T.; Physiopathology of Cushing's disease. Endocr. Rev. 4 (1983), 22.

(9) Liddle, G. W., W. F. Nicholson, D. P. Island, D. N. Orth, K. Abe, S. C. Lowder: Clinical and laboratory studies of ectopic humoral syndromes. Recent Progr. Hormone Res. 25 (1967) , 283.

(10) Loli, P., M. E. Berselli, M. Tagliaferri: Use of ketoconazole in the treatment of Cushing's syndrome. J. clin. Endocr. Metab. 63 (1986), 1365.

(11) Misbin, R. I., J. Canary, D. Willard: Aminoglutethimid in the treatment of Cushing's syndrome. J. clin. Pharmacol. 16 (1976), 645.

(12) Schteingart, D. E., H. S. Tsao, C. I. Tayler et al.: Sustained remission of Cushing's disease with mitotane and pituitary radiation. Ann. intern. Med. 92 (1980), 613.

(13) Sjöberg, H. E., M. Blombäck, P. O. Granberg: Thrombembolic complications, heparin treatment, and increase in coagulation factors in Cushing's syndrome. Acta med. scand. 199 (1976), 95. (14) Thoren, M., U. Adamson, H. E. Sjöberg,: Aminoglutethimid and metyrapone in the management of Cushing's syndrome. Acta endocr. (Kbh.) 109 (1985), 451 .

(15) Valimaki, M., R. Pelkonen, L. Porkka, A. Sivula, A. Kahri: Long-term results of adrenal surgery in patients with Cushing's syndrome due to adrenocortical adenoma. Clin. Endocr. 20 (1984), 229.

(16) Voigt, K. H., H. L. Fehm, R. Reck, E. F. Pfeiffer: Spontaneous and stimulated secretion of Quso-extractable immunoassayable ACTH in man. Klin. Wschr. 52 (1974), 516.

(17) Winkelmann, W., B. Allolio, D. Heesen, F. X. Hipp, D. Kaulen: Plasma cortisol, 11-deoxycortisol and ACTH in normal persons and in patients with adrenal insufficiency during metyrapone administration. Acta endocr. (Kbh.), Suppl. 234 (1980), 140.

(18) Zachmann, M., R. P. Gitzelmann, M. Zagalak, A. Prader: Effect of aminoglutethimid on urinary cortisol and cortisol metabolites in adolescents with Cushing's syndrome. Clin. Endocr. 7 (1977), 63.

(19) Zgliczynski, S., I. Kozlowicz, J. Wilczynak, B. Baranowska: Effects of cyproheptadine and metyrapone on ACTH and aldosterone concentrations in patients with Cushing's disease. A preliminary report. Materia med. pol. 12 (1980), 85.

Dr. M. Reincke, Dr. B. Allolio, Dr. Cornelia Jaursch-Hancke, Dr. D. Kaulen, Prof. Dr. W. Winkelmann

Medizinische Universitätsklinik II

5000 Köln 91, Ostmerheimer Str. 200 